\title{
Intrinsic Viscosity of Flexible Ring Polymers with Small Excluded Volume
}

\author{
Genzo Tanaka and Hiromi Yamakawa* \\ Department of Polymer Chemistry, Kyoto University, Kyoto, Japan.
}

(Received September 4, 1972)

\begin{abstract}
The intrinsic viscosity of flexible ring polymers with small excluded volume is calculated on the basis of the theory presented previously by Yamakawa and Tanaka for linear chains. The cubed viscosity-radius expansion factor is obtained as $\alpha_{\eta, \mathrm{r}^{3}}=1+1.21 z+\cdots$, so that $\alpha_{\eta}, \mathrm{r}^{3} / \alpha_{\eta}, 1^{3}=1+0.15 z+\cdots$, where the subscripts $\mathrm{r}$ and 1 refer to ring and linear forms, respectively, and $z$ is the well-known excluded-volume parameter. This result supports the prediction derived by Fukatsu and Kurata by an approximate perturbation method. Some comments on the Bloomfield-Zimm theory and the $\mathrm{Yu}-\mathrm{Fujita}$ theory are also given.

KEY WORDS Intrinsic Viscosity / Excluded-Volume Effect / Ring

Polymers / Perturbation Method /
\end{abstract}

In a previous paper, ${ }^{1}$ we have introduced exactly the excluded-volume effect into the Fixman-Pyun modification $^{2,3}$ of the ZimmHearst theory ${ }^{4-6}$ of the intrinsic viscosity of linear polymer chains by a perturbation method. The object of the present paper is to extend this method to the case of flexible ring polymers. The Zimm-Hearst theory for unperturbed chains without excluded volume involves two basic approximations: (1) preaveraging of the Oseen hydrodynamic interaction tensor, and (2) in our language, neglect of the coupling of the normal coordinates for hydrodynamic interactions. Within this framework, our previous first-order perturbation calculation of the viscosity-radius expansion factor $\alpha_{\eta}$ may be regarded as exact. This is also the case for the present calculation.

Originally, the theoretical investigation of the frictional properties of flexible rings was initiated by Bloomfield and $\mathrm{Zimm}^{7}$ with regard to biological interests. They introduced the excludedvolume effect into the $\mathrm{Zimm}$ theory ${ }^{4}$ of the intrinsic viscosity by the so-called $\varepsilon$-method of Peterlin. ${ }^{8}$ At about the same time, Fukatsu and Kurata ${ }^{9}$ treated the same problem by an approximate perturbation method, and obtained the result for $\alpha_{\eta}$ which was in conflict with the Bloomfield-Zimm theory. That is, $\alpha_{\eta, \mathrm{r}}>$

\footnotetext{
* To whom correspondence should be addressed.
}

$\alpha_{\eta, 1}$ in the former, while $\alpha_{\eta, \mathrm{r}}<\alpha_{\eta, 1}$ in the latter, where the subscripts $r$ and 1 refer to ring and linear chains, respectively. Thus, the problem was subsequently reexamined by $\mathrm{Yu}$ and Fujita following the procedure of Bloomfield and Zimm but with the use of a different circularity condition. This work has not been published but cited in the recent book by Yamakawa. ${ }^{10}$ However, we have recently noticed that the circularity condition adopted by $\mathrm{Yu}$ and Fujita is physically incorrect. For this reason we presume to re-reexamine the problem in the present paper.

\section{FORMULATION}

A large part of the formulation presented in a previous paper $^{1}$ for linear chains remains valid for the present problem. A minimum sketch is presented which is sufficient to understand the underlying physics and stress the differences between the problems for linear and ring polymers. We adopt the spring-and-bead model, i.e., a chain ring composed of $n+1$ identical Hooke's law springs joining $n+1$ identical beads. Each spring has a mean-square length $a^{2}$ and each bead has a friction constant $\zeta$ in a continuous solvent with viscosity coefficient $\eta_{0}$. Let $\mathbf{R}_{i}=\left(x_{i}, y_{i}, z_{i}\right)$ be the Cartesian coordinates of the $i$-th bead $(i=0,1,2, \cdots, n)$, and let $\left\{\mathbf{R}_{n+1}\right\}$ be the set of coordinates $\mathbf{R}_{0}, \mathbf{R}_{1}, \mathbf{R}_{2}, \ldots$, 
$\mathbf{R}_{n}$. Now we write the molecular distribution function $\boldsymbol{P}\left(\left\{\mathbf{R}_{n+1}\right\}, t\right)$ for the entire ring at time $t$ in the form,

$$
\boldsymbol{P}\left(\left\{\mathbf{R}_{n+1}\right\}, t\right)=\boldsymbol{P}_{0}\left(\left\{\mathbf{R}_{n+1}\right\}\right) \rho\left(\left\{\mathbf{R}_{n+1}\right\}, t\right)
$$

where $P_{0}$ is the equilibrium unperturbed distribution for the ring without excluded volume and may be written in the form,

$$
P_{0}=C_{0} \exp \left(-U_{0} / k T\right)
$$

with $U_{0}$ the spring potential of a quadratic form and $C_{0}$ the normalization constant. By the term "unperturbed," we mean that there is no excluded-volume effect, as usual. Further, we introduce the excluded-volume potential,

$$
W=k T \beta \sum_{i<j} \delta\left(\mathbf{R}_{i j}\right)
$$

where $\beta$ is the binary-cluster integral, or effective excluded volume, for a pair of beads, and $\mathbf{R}_{i j}\left(=\mathbf{R}_{j}-\mathbf{R}_{i}\right)$ is the separation between the $i$ th and $j$ th beads.

The function $\rho$ defined in eq 1 satisfies the differential equation, ${ }^{1-3,11}$

$$
\frac{\partial \rho}{\partial t}+L \rho=0
$$

with

$$
L=A_{0}+\beta A_{1}+L^{b}
$$

where the operator $L$ is split into three parts: the first an unperturbed self-adjoint operator $A_{0}$ with a scalar product defined with weighting function $\boldsymbol{P}_{0}$, the second $\beta A_{1}$ which involves the excluded-volume potential and vanishes in the unperturbed state, and the third $L^{b}$ which vanishes if the external force field (solvent flow) is absent. We choose as the basis set the eigenfunctions $\psi_{k}$ of the unperturbed free-draining time evolution operator $\boldsymbol{A}_{0}{ }^{0}$, i.e., the operator $\boldsymbol{A}_{0}$ without hydrodynamic interactions. The $\psi_{k}$ 's are products of various Hermite polynomials of the free-draining normal coordinates $\xi_{i}=\left(X_{i}, Y_{i}\right.$, $\left.Z_{i}\right)^{3}$ In matrix notation, we may write

$$
\mathbf{R}=\mathbf{Q \xi}
$$

where $\mathbf{R}=\left(\mathbf{R}_{0}, \mathbf{R}_{1}, \cdots, \mathbf{R}_{n}\right), \quad \xi=\left(\boldsymbol{\xi}_{0}, \boldsymbol{\xi}_{1}, \cdots, \boldsymbol{\xi}_{n}\right)$, and $\mathbf{Q}$ is an $(n+1) \times(n+1)$ orthogonal and symmetric transformation matrix. Then, $P_{0}$ becomes

$$
P_{0}=C_{0} \exp \left(-\sum_{k=1}^{n}{\sigma_{k}}^{2} \xi_{k}{ }^{2}\right)
$$

in the normal coordinates with $\sigma_{k}$ the scale factor, and $\rho$ may be expanded in terms of $\psi_{k}$.

Now, we assume the following original solvent flow field:

$$
\mathbf{v}^{0}=(\kappa y, 0,0)
$$

with $\kappa$ the time-independent velocity gradient. Further, we define the matrix elements of the operators $A_{0}$ and $A_{1}$ :

$$
\begin{aligned}
\lambda_{k}=\left\langle\psi_{k}, A_{0} \psi_{k}\right\rangle & =\int \boldsymbol{P}_{0} \psi_{k} A_{0} \psi_{k} \mathrm{~d}\left\{\boldsymbol{\xi}_{n+1}\right\} \\
A_{1, k l} & =\left\langle\psi_{k}, A_{1} \psi_{l}\right\rangle=\int \boldsymbol{P}_{0} \psi_{l k} \boldsymbol{A}_{1} \psi_{l} \mathrm{~d}\left\{\boldsymbol{\xi}_{n+1}\right\}
\end{aligned}
$$

As mentioned in the Introduction, we neglect the off-diagonal elements, $\left\langle\psi_{k}, A_{0} \psi_{l}\right\rangle$, of $A_{0}$. This is the diagonal approximation of Fixman and Pyun. When the hydrodynamic interaction is turned on, $A_{0}{ }^{0}$ becomes $A_{0}$. Then, the major effect is a modification of the diagonal elements, and the creation of the off-diagonal elements, or the coupling of the normal coordinates, is of minor significance. (Note that $\left\langle\psi_{k}, A_{0}{ }^{0} \psi_{l}\right\rangle=0$ for $k \neq l$.) When the excluded-volume interaction is further turned on, the terms $A_{1, k l}$ are added to the matrix elements of the time evolution operator. We take into account correctly all $A_{1, k l}$ 's through terms linear in $\beta$.

Then, the only states which can contribute to the first-order perturbation theory of the intrinsic viscosity $[\eta]$ for the flow given by eq 8 are of the types designated as $\phi_{(k)}$ and $\psi_{[k]}$ :

$$
\begin{gathered}
\phi_{(k)}=\frac{1}{2} H_{1}\left(\sigma_{k} X_{k}\right) H_{1}\left(\sigma_{k} Y_{k}\right) \\
\phi_{[k]}=2^{-3 / 2} H_{2}\left(\sigma_{k} Y_{k}\right)
\end{gathered}
$$

with $H_{p}$ the Hermite polynomials of degree $p$. The result reads

$$
\begin{aligned}
{[\eta]=} & \frac{N_{\mathrm{A}} k T}{M \eta_{0}} \sum_{k=1}^{n}\left[\frac{1}{\lambda_{(k)}}-\frac{\beta}{\lambda_{(k)}}\right. \\
& \left.\times\left(\sum_{l=1}^{n} \frac{A_{1,(k)(l)}}{\lambda_{(l)}}+\frac{\sqrt{2} A_{1,[k] 0}}{\lambda_{[k]}}\right)+\cdots\right]
\end{aligned}
$$

where $N_{\mathrm{A}}$ is the Avogadro number and $M$ the molecular weight of the polymer. Here and hereafter, the $k$ appearing as the product $k T$ is the Boltzmann constant and is not to be con- 
fused with the index $k$.

If we introduce the second approximation, i.e., preaveraging of the Oseen tensor, the matrix elements in eq 11 are found to be in the nondraining limit

$$
\begin{gathered}
\lambda_{(k)}=\lambda_{[k]}=\frac{4 k T}{3 \pi \eta_{0}} \sigma_{k}^{4} \sum_{\substack{i \\
i \neq j}} \sum_{j} Q_{k i} Q_{k j} F_{k}\left\langle R_{i j}{ }^{-1}\right\rangle_{0} \\
A_{1,(k)(l)}=\frac{4 k T}{3 \pi \eta_{0}}{\sigma_{k}}^{2} \sigma_{l}{ }^{2} \sum_{\substack{i \\
i \neq j \neq t}} \sum_{\substack{j \\
i \neq j}} Q_{k i} U_{k l}\left\langle R_{i j}{ }^{-1}\right\rangle_{0} \\
A_{1,[k]_{0}}=\frac{\sqrt{2 k T}}{3 \pi \eta_{0}}{\sigma_{k}}^{2} \sum_{\substack{i \\
i \neq j \neq t}} \sum_{\substack{j \\
i \neq j}} Q_{k i} V_{k}\left\langle R_{i j}{ }^{-1}\right\rangle_{0}
\end{gathered}
$$

with

$$
\begin{gathered}
F_{k}=\left\langle\left(\sum_{m} Q_{k m} y_{m}\right)^{2}\right\rangle_{0} \\
U_{k l}=\left\langle\left(\sum_{m} Q_{k m} y_{m}\right)\left(\sum_{m} Q_{l m} y_{m}\right)\left(\sum_{m} Q_{l m} x_{m}\right) \delta_{x}\left(\mathbf{R}_{t j}\right)\right\rangle_{0} \\
V_{k}=\left\langle\left(\sum_{m} Q_{k m} y_{m}\right) \delta_{y}\left(\mathbf{R}_{t j}\right\rangle_{0}\right.
\end{gathered}
$$

where \langle\rangle$_{0}$ designates an equilibrium unperturbed average, $Q_{k j}$ are the elements of $\mathbf{Q}$, and

$$
\delta_{x}\left(\mathbf{R}_{t j}\right)=\frac{\partial}{\partial x_{j}} \delta\left(\mathbf{R}_{j}-\mathbf{R}_{t}\right)
$$

The above formulation is formally valid for both linear and ring polymers, and we must now consider the differences between the two forms, which appear in $Q_{k j}, \sigma_{k}$, and $\left\langle R_{i j}{ }^{-1}\right\rangle_{0}$. For ring polymers, these are given by

$$
\begin{aligned}
Q_{k j} & =\left(\frac{2}{n}\right)^{1 / 2} \cos \left(\frac{2 k j \pi}{n}\right) \text { for } k=0,1, \ldots, \frac{n}{2} \\
& =\left(\frac{2}{n}\right)^{1 / 2} \sin \left(\frac{2 k j \pi}{n}\right) \text { for } k=\frac{n}{2}+1, \cdots, n
\end{aligned}
$$

$$
\begin{gathered}
{\sigma_{k}}^{2}=\frac{3 m_{k}}{2 a^{2}} \\
m_{k}=4 \sin ^{2}\left(\frac{k \pi}{n}\right) \\
\left.{R_{i j}}^{-1}\right\rangle_{0}=\left(\frac{6}{\pi}\right)^{1 / 2} \frac{n^{1 / 2}}{|i-j|^{1 / 2}(n-|i-j|)^{1 / 2} a}
\end{gathered}
$$

Without loss of generality, we have assumed that $n$ is even. Further, note that $m_{k}$ are the Rouse eigenvalues for the ring, and that there are the symmetry properties,

$$
\begin{aligned}
& m_{k}=m_{n-k} \\
& {\sigma_{k}}^{2}=\sigma_{n-k}{ }^{2}
\end{aligned}
$$

\section{CALCULATIONS}

The steps involved in the calculation are: (1) evaluation of the averages $F_{k}, U_{k l}$, and $V_{l}$ given by eq 13, (2) summations over $i, j$, and $t$ in eq 12, and (3) summations over $k$ and $l$ in eq 11.

First, the averages $F_{k}, U_{k l}$, and $V_{k}$ can be evaluated using the Gaussian distribution $\boldsymbol{P}_{\mathrm{r}}\left(\boldsymbol{\Phi}_{1}\right.$, $\left.\Phi_{2}, \Phi_{3}\right)$ of the vectors,

$$
\begin{gathered}
\Phi_{1}=\sum_{j} Q_{k j} \mathbf{R}_{j} \\
\Phi_{2}=\sum_{j} Q_{l j} \mathbf{R}_{j} \\
\Phi_{3}=\mathbf{R}_{t j}
\end{gathered}
$$

the subscript $\mathrm{r}$ on $\boldsymbol{P}$ referring to the ring polymer, as before. This distribution can be derived from the distribution $\boldsymbol{P}_{1}\left(\Phi_{1}, \Phi_{2}, \Phi_{3}, \Phi_{4}\right)$ with $\Phi_{4}=\mathbf{R}_{0 \mathrm{n}}$ for the linear chain as follows,

$$
\begin{aligned}
& P_{\mathrm{r}}\left(\Phi_{1}, \Phi_{2}, \Phi_{3}\right) \\
& =\frac{P_{1}\left(\Phi_{1}, \Phi_{2}, \Phi_{3}, \Phi_{4}=0\right)}{\int P_{1}\left(\Phi_{1}, \Phi_{2}, \Phi_{3}, \Phi_{4}=0\right) \mathrm{d} \Phi_{1} \mathrm{~d} \Phi_{2} \mathrm{~d} \Phi_{3}}
\end{aligned}
$$

In the coordinate system where the molecular center of mass is taken as origin, by the use of the Wang-Uhlenbeck-Fixman theorem ${ }^{12,13}$ the Gaussian distribution $P_{1}$ is found to be

$$
\begin{aligned}
& \boldsymbol{P}_{1}\left(\boldsymbol{\Phi}_{1}, \cdots, \boldsymbol{\Phi}_{4}\right)=\left(\frac{3}{2 \pi a^{2}}\right)^{6} c^{-3 / 2} \\
& \quad \times \exp \left(-\frac{3}{2 a^{2} c} \sum_{p=1}^{4} \sum_{q=1}^{4} c^{p q} \boldsymbol{\Phi}_{p} \cdot \boldsymbol{\Phi}_{q}\right)
\end{aligned}
$$

where $c^{p q}$ is the cofactor of the element $c_{p q}$ of the $4 \times 4$ determinant $c$, whose elements are given in the Appendix. Then the results are

$$
\begin{aligned}
& F_{k}=\frac{1}{3} a^{2}\left(c_{11} c_{44}-c_{14}{ }^{2}\right) c_{44}{ }^{-1} \\
& U_{k l}=-\frac{1}{3} a^{2}\left(\frac{3}{2 \pi a^{2}}\right)^{3 / 2}\left\{c_{11} c_{44}{ }^{3 / 2}\left(c_{13} c_{44}-c_{14} c_{34}\right)\right. \\
& \times\left(c_{33} c_{44}-c_{34}{ }^{2}\right)^{-5 / 2} \delta_{k l}-c_{44}{ }^{3 / 2}\left(c_{23} c_{44}-c_{24} c_{34}\right) \\
& \times\left[c_{12}\left(c_{34}{ }^{2}-c_{33} c_{44}\right)+c_{13}\left(c_{23} c_{44}-c_{24} c_{34}\right)\right. \\
&\left.\left.+c_{14}\left(c_{24} c_{33}-c_{23} c_{34}\right)\right]\left(c_{33} c_{44}-c_{34}{ }^{2}\right)^{-7 / 2}\right\} \\
& V_{l}=-\left(\frac{3}{2 \pi a^{2}}\right)^{3 / 2} c_{44}{ }^{3 / 2}\left(c_{14} c_{34}-c_{13} c_{44}\right)\left({ }_{33} c_{44}-c_{34}{ }^{2}\right)^{-5 / 2}
\end{aligned}
$$

When $k=l$, the subscript 2 should be replaced by the subscript 1 in $U_{k l}$, since then $\Phi_{2}=\Phi_{1}$. 
Now, noting the symmetry property, $\lambda_{(k)}=$ $\lambda_{(n-k)}$, it is convenient to define:

$$
\begin{gathered}
\bar{\lambda}_{(k)}=\frac{1}{2}\left(\lambda_{(k)}+\lambda_{(n-k)}\right) \quad\left(\bar{\lambda}_{(k)}=\lambda_{(k)}\right) \\
\bar{A}_{1,(k)(l)}=A_{1,(k)(l)}+A_{1,(k)(n-l)}+A_{1,(n-k)(l)} \\
+A_{1,(n-k)(n-l)} \\
\bar{A}_{1,[k] 0}=\frac{1}{2}\left(A_{1,[k] 0}+A_{1,[n-k] 0}\right)
\end{gathered}
$$

Then eq 11 becomes

$$
\begin{aligned}
{[\eta]_{\mathrm{r}}=} & \frac{2 N_{\mathrm{A}} k T}{M_{\eta_{0}}} \sum_{k=1}^{n / 2}\left[\frac{1}{\bar{\lambda}_{(k)}}-\frac{\beta}{\bar{\lambda}_{(k)}}\left(\sum_{l=1}^{n / 2} \frac{\bar{A}_{1,(k)(l)}}{\bar{\lambda}_{(l)}}\right.\right. \\
& \left.\left.+\frac{\sqrt{2} \bar{A}_{1,[k] 0}}{\bar{\lambda}_{(k)}}\right)+\cdots\right]
\end{aligned}
$$

We replace the sums over $i, j$, and $t$ by integrals in eq 12 , and obtain

$$
\begin{gathered}
\bar{\lambda}_{(k)}=\frac{16 k T}{3 \eta_{0}}\left(\frac{3}{2 \pi a^{2}}\right)^{3 / 2} n^{-3 / 2} I_{1}(k) \\
\bar{A}_{1,(k)(l)}=-\frac{64 k T}{3 \eta_{0} n}\left(\frac{3}{2 \pi a^{2}}\right)^{3}\left[\delta_{k l} I_{2}(k)-I_{3}(k, l)\right] \\
\bar{A}_{1,[k] 0}=-\frac{32 \sqrt{2} k T}{3 \eta_{0} n}\left(\frac{3}{2 \pi a^{2}}\right)^{3} I_{2}(k)
\end{gathered}
$$

where

$$
\begin{aligned}
& I_{1}(k)=\frac{1}{2} k^{2} \pi^{2} \int_{-1}^{1} \int_{-1}^{1}|x-y|^{-1 / 2}(2-|x-y|)^{-1 / 2} \\
& \times \cos (y-x) k \pi \mathrm{d} x \mathrm{~d} y \\
& I_{2}(k)=\int_{-1}^{1} \int_{-1}^{1} \int_{-1}^{1}|x-y|^{-1 / 2}(2-|x-y|)^{-1 / 2} \mid y \\
& -\left.z\right|^{-5 / 2}(2-|y-z|)^{-5 / 2} \sin \frac{1}{2}(y-z) k \pi \\
& \times \sin \frac{1}{2}[2(x-y)+(y-z)] k \pi \mathrm{d} x \mathrm{~d} y \mathrm{~d} z \\
& I_{3}(k, l)=\frac{8}{l^{2} \pi^{2}} \int_{-1}^{1} \int_{-1}^{1} \int_{-1}^{1}|x-y|^{-1 / 2}(2-\mid x \\
& -y \mid)^{-1 / 2}|y-z|^{-7 / 2}(2-|y-z|)^{-7 / 2} \\
& \times \sin \frac{1}{2}(y-z) k \pi \sin \frac{1}{2}[2(x-y)+(y-z)] k \pi \\
& \times \sin ^{2} \frac{1}{2}(y-z) l \pi \mathrm{d} x \mathrm{~d} y \mathrm{~d} z
\end{aligned}
$$

The integral $I_{1}(k)$ can be calculated analytically as

$$
I_{1}(k)=(-1)^{k} k^{2} \pi^{3} J_{0}(k \pi)
$$

where $J_{0}$ is the Bessel function of the zeroth order defined by

$$
J_{0}(k \pi)=\frac{2}{\pi} \int_{0}^{1} \frac{\cos k \pi x}{\left(1-x^{2}\right)^{1 / 2}} \mathrm{~d} x
$$

with the asymptotic form $(-1)^{k} / k^{1 / 2}$ for large $k$. The integrals $I_{2}$ and $I_{3}$ can be reduced to double integrals but cannot be evaluated analytically. These double integrals may be evaluated by the use of a computer.

Thus, the final equation may be written in the form,

$$
[\eta]_{\mathrm{r}} /[\eta]_{\theta, \mathrm{r}} \equiv \alpha_{\eta, \mathrm{r}}^{3}=1+C_{1, \mathrm{r}} z+\cdots
$$

with

$$
\begin{aligned}
{[\eta]_{\theta, \mathrm{r}} } & =\frac{2 N_{\mathrm{A}} k T}{M \eta_{0}} \sum_{k=1}^{n / 2}\left(\bar{\lambda}_{(k)}\right)^{-1} \\
& =12^{3 / 2} \Phi_{0, \mathrm{r}}\left\langle S^{2}\right\rangle_{0, \mathrm{r}}{ }^{3 /} M . \\
\Phi_{0, \mathrm{r}} & =\frac{\sqrt{6}}{12} \pi^{3 / 2} N_{\mathrm{A}} S_{0} \\
C_{1, \mathrm{r}} & =4 S_{0}^{-1}\left(2 S_{1}-S_{2}-S_{3}\right)
\end{aligned}
$$

where $[\eta]_{\theta, \mathrm{r}},\left\langle S^{2}\right\rangle_{0, \mathrm{r}}\left(=\frac{1}{2}\left\langle S^{2}\right\rangle_{0,1}=n a^{2} / 12\right)$, and $\Phi_{0, \mathrm{r}}$ are the intrinsic viscosity, mean-square radius of gyration, and viscosity constant of the unperturbed ring, respectively, $z$ is the well-known excluded-volume parameter defined by

$$
z=\left(3 / 2 \pi a^{2}\right)^{3 / 2} \beta n^{1 / 2}
$$

and $S_{0}$ to $S_{3}$ are sums defined by

$$
\begin{aligned}
S_{0} & =\sum_{k=1}^{n / 2}\left[I_{1}(k)\right]^{-1} \\
S_{1} & =\sum_{k=1}^{n / 2} G_{1}(k) \\
S_{2} & =\sum_{k=1}^{n / 2} G_{2}(k, k) \\
S_{3} & =\sum_{\substack{k=1 \\
k / 2}}^{n / 2} G_{l=1}^{n / 2} G_{2}(k, l)
\end{aligned}
$$

with

$$
\begin{gathered}
G_{1}(k)=I_{2}(k) /\left[I_{1}(k)\right]^{2} \\
G_{2}(k, l)=I_{3}(k, l) / I_{1}(k) I_{1}(l)
\end{gathered}
$$

Our problem which remains is to evaluate these sums. We evaluate $S_{0}$ by perfoming summation with the use of the values of the Bessel function from $k=1$ to $k=20$ and of the EulerMaclaurin summation formula for $k \geq 21$ with the asymptotic form $I_{1}(k)=\pi^{2} k^{2 / 2}$. The result is

$$
S_{0}=0.2708
$$

so that

$$
\Phi_{0, \mathrm{r}}=1.854 \times 10^{23}
$$


The corresponding value of $1.85 \times 10^{23}$ for $\Phi_{0, \mathrm{r}}$ has already been obtained by Bloomfield and Zimm $^{7}$ and by Fukatsu and Kurata. ${ }^{9}$

The evaluation of the sums $S_{1}$ to $S_{3}$ is completely analogous to that of the corresponding sums for linear chains, ${ }^{1}$ and we do not reproduce the details. We merely note that the steps consist of evaluating double and triple integrals on a FACOM 230-60 digital computer at Kyoto University and of applying the Euler-Maclaurin summation formula. The necessary asymptotic forms found from the values of the integrals are

$$
\begin{gathered}
G_{1}(k)=0.03380 k^{-2} \\
G_{2}(k, k)=0.007200 k^{-3} \\
\sum_{\substack{l=1 \\
\neq \neq k}}^{n / 2} G_{2}(k, l)=0.03480 k^{-3 / 2}-0.02560 k^{-5 / 2} \quad(k \geq 21)
\end{gathered}
$$

The results thus obtained are

$$
\begin{aligned}
& S_{1}=0.07980 \\
& S_{2}=0.02446 \\
& S_{3}=0.05292
\end{aligned}
$$

With these values, we finally obtain for the coefficient $C_{1, \mathrm{r}}$ in eq 30

$$
C_{1, \mathrm{r}}=1.21
$$

\section{DISCUSSION}

For linear chains, we have already obtained the value of 1.06 for the coefficient $C_{1,1}$ corresponding to eq $41 .^{1}$ Combining these two results, we have

$$
\alpha_{\eta, \mathrm{r}}{ }^{3} / \alpha_{\eta, 1}{ }^{3}=1+0.15 z+\cdots,
$$

so that $\alpha_{\eta, \mathrm{r}}$ is greater than $\alpha_{\eta, 1}$ provided $z$ is positive. Now, we note that for the expansion factor of the radius of gyration, there has been derived the expansion, ${ }^{14}$

$$
\alpha_{\mathrm{s}, \mathrm{r}}{ }^{2} / \alpha_{\mathrm{s}, 1}{ }^{2}=1+0.295 z+\cdots,
$$

which is the exact first-order perturbation theory result. Both results may be regarded as arising from the fact that ring formation gives rise to an increase in segment density, thereby leading to greater excluded-volume effects. In the near future, it will be possible to compare these predictions with experimental results for ring polystyrenes. ${ }^{15}$

As already discussed, we have taken into account all matrix elements of the time evolution operator for excluded-volume interactions but only the diagonal elements for hydrodynamic interactions. If the off-diagonal elements are ignored also for excluded-volume interactions, we obtain for the diagonal approximation of $C_{1, \mathrm{r}}$

$$
C_{1, \mathrm{r}}^{(D)}=4 S_{0}^{-1}\left(S_{1}-S_{2}\right)=0.817
$$

This value is very close to the corresponding value of 0.810 previously obtained for linear chains. ${ }^{1}$ Therefore, the difference between $C_{1, \mathrm{r}}$ and $C_{1,1}$ is seen to arise mainly from the alteration of the off-diagonal elements by ring formation.

Finally, some discussion on other theories of $\alpha_{\eta, \mathrm{r}}$ is appropriate. For the perturbed ring with excluded volume, Bloomfield and $\mathrm{Zimm}^{\text {? }}$ have assumed as a starting equation

$$
\left\langle R_{i j}{ }^{2}\right\rangle_{\mathrm{r}}=\frac{|i-j|^{1+\varepsilon}(n-|i-j|)^{1+\varepsilon}}{|i-j|^{1+\varepsilon}+(n-|i-j|)^{1+\varepsilon}} a^{2}
$$

where $\varepsilon$ is a small positive number representing the excluded-volume effect. This equation may be derived from the following consideration. Suppose that the ring is cut off at the $i$ th and $j$ th beads into two linear chains composed of $|i-j|$ and $n-|i-j|$ beads. We designate their mean-square end-to-end distances by $\left\langle\boldsymbol{R}_{1}{ }^{2}\right\rangle_{1}$ and $\left\langle\boldsymbol{R}_{2}{ }^{2}\right\rangle_{1}$, respectively. Then, in the unperturbed state, there holds exactly the relation,

$$
\left\langle\boldsymbol{R}_{i j}{ }^{2}\right\rangle_{\mathrm{r}}^{-1}=\left\langle\boldsymbol{R}_{1}{ }^{2}\right\rangle_{1}{ }^{-1}+\left\langle\boldsymbol{R}_{2}{ }^{2}\right\rangle_{1}^{-1}
$$

Now, if we assume that this equation is valid also for the perturbed ring, and write $\left\langle\boldsymbol{R}_{1}{ }^{2}\right\rangle_{1}=$ $|i-j|^{1+\varepsilon} a^{2}$ and $\left\langle R_{2}{ }^{2}\right\rangle_{1}=(n-|i-j|)^{1+\varepsilon} a^{2}$ by an application of the $\varepsilon$-method, then eq 45 is obtained. However, eq 45 leads to the results, $\alpha_{\mathrm{s}, \mathrm{r}}<\alpha_{\mathrm{s}, 1}$ and $\alpha_{\eta, \mathrm{r}}<\alpha_{\eta, 1}$, which are in conflict with those given by eq 42 and 43 . On the other hand, $\mathrm{Yu}$ and Fujita ${ }^{10}$ have assumed

$$
\left\langle R_{i j}{ }^{2}\right\rangle_{\mathrm{r}}=\frac{|i-j|^{1+\varepsilon}\left(n^{1+\varepsilon}-|i-j|^{1+\varepsilon}\right)}{n^{1+\varepsilon}} a^{2}
$$

instead of eq 45 . This equation gives the results, $\alpha_{\mathrm{s}, \mathrm{r}}>\alpha_{\mathrm{s}, 1}$ and $\alpha_{\eta, \mathrm{r}} \lesssim \alpha_{\eta, 1}$, the former being consistent with eq 43 . However, eq 47 does not 
satisfy the simple physical requirement that $\left\langle\boldsymbol{R}_{i j}{ }^{2}\right\rangle_{\mathrm{r}}$ be invariant to the exchange of $|i-j|$ and $n-|i-j|$, the numbers of beads in $R_{1}$ and $R_{2}$. Thus, we may conclude that it is very dangerous to draw precise quantitative conclusions for the excluded-volume effect by an application of the $\varepsilon$-method. Fukatsu and Kurata ${ }^{9}$ have used an approximate perturbation method to derive

$$
\alpha_{\eta, \mathrm{r}}{ }^{3} / \alpha_{\eta, 1}{ }^{3}=1+0.31 z+\cdots
$$

This prediction is consistent with but less rigorous than the present result given by eq 42 . Imai $^{16}$ has also investigated the same problem though he did not obtain concrete numerical results.

\section{APPENDIX}

In this appendix, we give the matrix elements $c_{p q}$ in eq 22. They depend on the ranges of the indices $k$ and $l$, and there are four possible cases to be considered.

(1) For $1 \leq k, l \leq n / 2, c_{p q}$ are given by

$$
\begin{gathered}
c_{11}=m_{k}^{-1}, \quad c_{22}=m_{l}^{-1}, \quad c_{33}=|j-t|, \quad c_{44}=n \\
c_{12}=c_{21}=c_{14}=c_{41}=c_{24}=c_{42}=0 \\
c_{13}=c_{31}=m_{k}^{-1}\left(Q_{l j}-Q_{k t}\right) \\
c_{23}=c_{32}=m_{l}^{-1}\left(Q_{l j}-Q_{l t}\right) \\
c_{34}=c_{43}=j-t .
\end{gathered}
$$

(2) For $k \leq n / 2<l$, the elements $c_{p q}$ with $p \neq$ 2 and $q \neq 2$ are given by the corresponding elements of eq $A 1$ and the remaining elements possessing the subscripts $p 2$ and 22 are given by

$$
\begin{gathered}
c_{22}=3 m_{l}^{-1}, \quad c_{12}=c_{21}=0 \\
c_{23}=c_{32}=2 m_{l}^{-1}\left[Q_{l j}-Q_{l t}-\left(m_{l} / 2 n\right)^{1 / 2}(j-t)\right] \\
c_{24}=c_{42}=-\left(2 n / m_{l}\right)^{1 / 2}
\end{gathered}
$$

Note that these elements are associated with the vector $\Phi_{2}$.

(3) For $l \leq n / 2<k$, all $c_{p q}$ are produced from the elements in Case (2) by exchanging the subscripts $l$ and $k$ for the subscripts 2 and $l$, respectively.

(4) For $n / 2<k, l \leq n$, the elements possessing the subscripts 1 and 2 are given by the corresponding elements in Cases (3) and (2), respectively, except that

$$
c_{12}=c_{21}=\left(2+\delta_{k l}\right) /\left(m_{k} m_{l}\right)^{1 / 2}
$$

and the remaining elements are given by the corresponding elements of eq A1.

\section{REFERENCES}

1. H. Yamakawa and G. Tanaka, J. Chem. Phys., 55, 3188 (1971).

2. M. Fixman, ibid., 42, 3831 (1965).

3. C. W. Pyun and M. Fixman, ibid., 42, 3838 (1965); 44, 2107 (1966).

4. B. H. Zimm, ibid., 24, 269 (1956).

5. B. H. Zimm, G. M. Roe, and L.F. Epstein, ibid., 24, 279 (1956).

6. J. E. Hearst, ibid., 37, 2547 (1962).

7. V. Bloomfield and B. H. Zimm, ibid., 44, 315 (1966).

8. A. Peterlin, ibid., 23, 2464 (1955).

9. M. Fukatsu and M. Kurata, ibid., 44, 4539 (1966).

10. H. Yamakawa, "Modern Theory of Polymer Solutions," Harper and Row, Publishers, New York, N.Y., 1971, Chap. VI.

11. Fixman, J. Chem. Phys., 45, 785, 793 (1966).

12. M. C. Wang and G. E. Uhlenbeck, Rev. Mod. Phys., 17, 323 (1945).

13. M. Fixman, J. Chem. Phys., 23, 1656 (1955).

14. E. F. Casassa, J. Polym. Sci., Part A, 3, 605 (1965).

15. $\mathrm{H} . \mathrm{Yu}$, private communication.

16. S. Imai, J. Chem. Phys., 51, 1732 (1969). 